\title{
Two-dimensional theory of coherent synchrotron radiation with transient effects
}

\author{
Yunhai Cai \\ SLAC National Accelerator Laboratory, 2575 Sand Hill Road, Menlo Park, California 94205, USA
}

(Received 23 April 2021; accepted 14 June 2021; published 28 June 2021)

\begin{abstract}
A two-dimensional theory of coherent synchrotron radiation for a bending magnet with finite length is developed as an extension to the one-dimensional theory. On axis, the transient longitudinal wakes agree exactly to those in the one-dimensional theory. Off axis, the deviations are small but noticeable. The theory includes the transient centrifugal and centripetal forces after electron bunch enters and then exits the magnet. However, the transverse wakes are an order of magnitude smaller than the longitudinal ones.
\end{abstract}

DOI: $10.1103 /$ PhysRevAccelBeams.24.064402

\section{INTRODUCTION}

Historically, coherent synchrotron radiation (CSR) in electron storage rings was first studied in an unpublished report [1] by Schwinger, who found that the coherent radiation power was suppressed by the parallel metal walls. His work essentially was a calculation of the real part of the longitudinal impedance in the frequency domain. The work was extended to include both real and imaginary parts of the impedance in toroidal chamber by Warnock and Morton [2]. Correspondingly in the time domain, the longitudinal wake with shielding from the parallel plates was derived later by Murphy et al., [3]. Based on the wake, the theory [4] of predicting the threshold of the microwave instability with the scaled bunch current and the shielding parameter was developed. The instability prevents the bunch from having an adequate current and shorter than a few millimeters.

In single-pass and linac-based accelerators, the bunch length can be much shorter than that in rings. However, CSR remains an important limitation to advance the brightness of the electron beam. It causes microbunching $[5,6]$, and increases energy spread and emittance. Because of the short bunch, the shielding does not play an important role as in the rings. Instead, the wake in free space [3,7] dominantly degrades the beam quality in the bunch compressors.

The collective beam dynamics in the compressors can be well described by the one-dimensional (1D) theory of CSR. Its development can be outlined in three main stages. The first step was the numerical computation of the transient

Published by the American Physical Society under the terms of the Creative Commons Attribution 4.0 International license. Further distribution of this work must maintain attribution to the author(s) and the published article's title, journal citation, and DOI. wakes due to a finite length of bending magnet by Dohlus and Limberg [8], who found that the transients became important when the length of the bending magnet was at the same order of the overtaking length [9] $L_{0}=\left(24 \sigma_{z} \rho^{2}\right)^{1 / 3}$, where $\sigma_{z}$ is the bunch length and $\rho$ is the bending radius. The second step was the analytical formulation by Saldin et al., [10]. The final step was the simplification at the ultrarelativistic limit by Stupakov and Emma [11]. The 1D theory is simple and elegant. It has been implemented in many simulation codes, such as ELEGANT [12] and routinely used for the design of bunch compression. The simulations are in good agreement with the experimental measurements [13] for the peak currents below $5 \mathrm{kA}$.

Despite the success of the 1D theory, it is not yet a complete theory because of the lack of an explanation of why the transverse force can be ignored. The importance of the centrifugal force was first emphasized by Talman [14]. It was carefully analyzed by Lee [15], who showed that it was canceled by a potential term due to the energy change within a coasting beam. For a bunched beam, Derbenev and Shiltsev [9] found a similar cancellation but with a residual centripetal wake. This centripetal force is neglected in the 1D theory.

Moreover, the 1D theory requires "regularization", namely subtracting a singular longitudinal space-charge force in free space. Although, the reason for introducing the regularization was well articulated [10], it remains unsatisfied from a theoretical viewpoint because the theory itself is classical.

From these prospectives, we will begin a journey of developing a two-dimensional theory with transients as an extension to the 1D theory. Naturally, the two-dimensional theory [16] in steady state is a good base to start. We will continue from Sec. II to $\mathrm{V}$ with the four corresponding cases, labeled (A), (B), (C), and (D) in the 1D theory [10]. In Sec. VI, we will provide some numerical examples for illustration. Finally, we will conclude in Sec. VII. 
In classical electrodynamics, the electric and magnetic fields generated by an electron moving on a curved orbit can be calculated with the Lienard-Wiechert formula [17],

$$
\begin{aligned}
\vec{E}=e\left[\frac{\hat{n}-\vec{\beta}}{\gamma^{2}(1-\vec{\beta} \cdot \hat{n})^{3} R^{2}}\right]_{\mathrm{ret}}+\frac{e}{c}\left[\frac{\hat{n} \times\{(\hat{n}-\vec{\beta}) \times \dot{\vec{\beta}}\}}{(1-\vec{\beta} \cdot \hat{n})^{3} R}\right]_{\mathrm{ret}}, \\
\vec{B}=\hat{n} \times \vec{E},
\end{aligned}
$$

where $\hat{n}=\vec{R} / R$ and $\vec{R}$ is the vector, starting from the source point and ending at the observation point. The source point is where the radiation is emitted at the retarded time,

$$
t^{\prime}=t-\frac{R}{c}
$$

and $t$ is the time when the observation is made. $\vec{\beta}$ and $\dot{\vec{\beta}}$ are the velocity in unit of the speed of light and acceleration of the electron at the retarded time, respectively.

In the Lienard-Wiechert formula, the first term suppressed by $1 / \gamma^{2}$ is called the space charge and the second term driven by the acceleration is often called CSR.

\section{CASE (A)}

First, we consider the case when the source point is in the free space before the entry of the magnet and the observation point is inside the magnet as illustrated in Fig. 1. Here, we use the arc length $s$ instead time $t$ as independent variable.

Given the velocity $v$ of a particle, the path length between point $s$ and $s^{\prime}$ can be written as

$$
s-s^{\prime}=v\left(t-t^{\prime}\right)+\left(z-z^{\prime}\right)
$$

where $z=s-v t$ and $z^{\prime}=s^{\prime}-v t^{\prime}$ are the longitudinal positions of the particle at time $t$ and $t^{\prime}$, respectively. As illustrated in Fig. 1, it is also given by

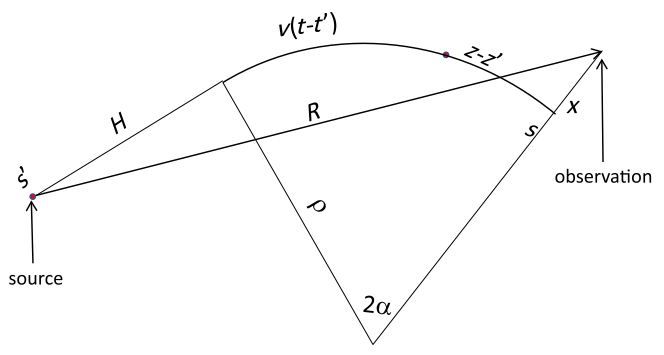

FIG. 1. A source particle moves first on straight line and then on circular orbit with a bending radius $\rho$ while the observation point is inside the magnet.

$$
s-s^{\prime}=H+2 \alpha \rho,
$$

where $H$ is the distance between the source point and the entry of the magnet and $\alpha$ is the half of the angular position inside the magnet. Substituting $s-s^{\prime}$ in Eq. (5) and $t-t^{\prime}$ in Eq. (3) into Eq. (4), we obtain the retarded condition in terms of the retarded distance $\eta=H / \rho$,

$$
\xi=\alpha+\frac{\eta-\beta \kappa}{2},
$$

where $\kappa=R / \rho$ and $\xi=\left(z-z^{\prime}\right) / 2 \rho$. Applying trigonometry involving three neighboring triangles, we find explicitly

$\kappa=\sqrt{\eta^{2}+\chi^{2}+4(1+\chi) \sin ^{2} \alpha+2 \eta(1+\chi) \sin 2 \alpha}$,

where $\chi=x / \rho$.

Given $\alpha, \chi$, and $\xi$, the retarded condition Eq. (6) can be rewritten in terms of a quadratic equation of $\eta$,

$$
\begin{aligned}
& \frac{1}{4}\left(1-\beta^{2}\right) \eta^{2}+\left[\alpha-\xi-\frac{1}{2} \beta^{2}(1+\chi) \sin 2 \alpha\right] \eta \\
& \quad+\left[\alpha^{2}-2 \alpha \xi+\xi^{2}-\frac{\beta^{2}}{4} \chi^{2}-\beta^{2}(1+\chi) \sin ^{2} \alpha\right]=0,
\end{aligned}
$$

where $\eta$ is a root of the quadratic equation. Comparing it to the general equation, $a \eta^{2}+b \eta+c=0$, the analytical solution is given by

$$
\eta=\frac{-b+\sqrt{b^{2}-4 a c}}{2 a} .
$$

Note that $\eta$ becomes zero when $c=0$ or $\xi=$ $\alpha-\frac{\beta}{2} \sqrt{\chi^{2}+4(1+\chi) \sin ^{2} \alpha}$, which defines the upper bound of the longitudinal integration for bunch wakes.

On the other hand, the retarded distance $\eta$ also can be found numerically with a direct Newton searching using Eq. (6). Numerical examples are shown Fig. 2 along with the analytical solutions from Eq. (8). Since the agreements are perfect, we will use the analytical solutions in the rest of the paper. It is worth noting that the particles with different horizontal positions enter the magnet in their corresponding longitudinal positions. The effects will be taken into account when we perform the integrations of computing the bunch wakes.

Since the source is in the free space, the electron is moving on a straight line with a constant speed and therefore no acceleration. As a result, only the first term or the space-charge term in Eq. (1) contributes to the fields. After straightforward but tedious algebra of solving three triangles consecutively, we derive their nonvanishing components, 


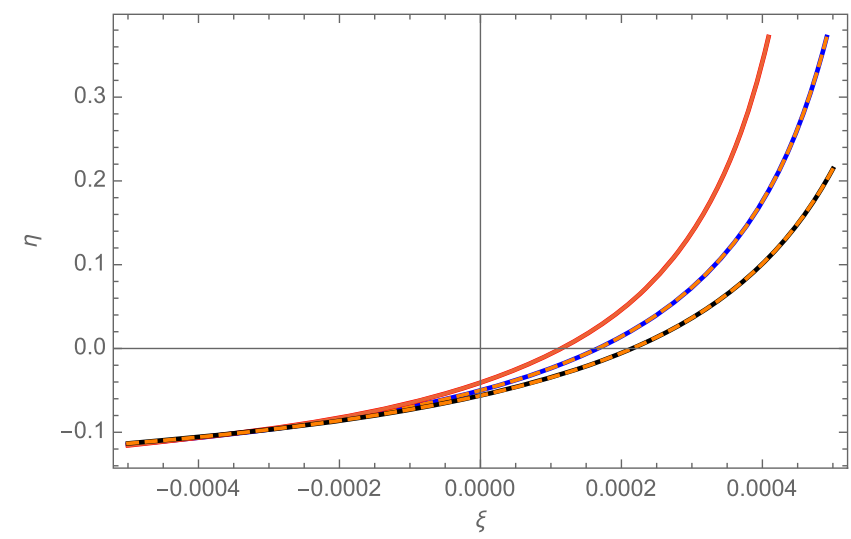

FIG. 2. Comparison of the numerical (solid line) and analytical (dashed orange) solutions of $\eta$ as a function of $\xi$ with $\alpha=0.1$, $\chi=0$ (blue), 0.001 (red), -0.001 (black), and $\gamma=5000$.

$$
\begin{array}{r}
E_{x}=\frac{e[1+\chi-\cos 2 \alpha+(\eta-\beta \kappa) \sin 2 \alpha]}{\gamma^{2} \rho^{2}\{\kappa-\beta[\eta+(1+\chi) \sin 2 \alpha]\}^{3}}, \\
E_{s}=\frac{e[\sin 2 \alpha+(\eta-\beta \kappa) \cos 2 \alpha]}{\gamma^{2} \rho^{2}\{\kappa-\beta[\eta+(1+\chi) \sin 2 \alpha]\}^{3}}, \quad \text { and } \\
B_{y}=\frac{e \beta[(1+\chi) \cos 2 \alpha-1]}{\gamma^{2} \rho^{2}\{\kappa-\beta[\eta+(1+\chi) \sin 2 \alpha]\}^{3}},
\end{array}
$$

where the retarded distance $\eta$ is given by Eq. (8), through which the longitudinal dependency enters.

Given a design momentum $p_{0}$, the relative change $\delta=\left(p-p_{0}\right) / p_{0}$ per unit length is given by, $\delta=e E_{s} / c p_{0} \beta$, assuming the particles moving in the longitudinal velocity $\beta$. For a bunch of particles moving uniformly, using the longitudinal electric field $E_{s}$ between two particles in Eq. (10) as Green's function, we have

$$
\dot{\delta}=\frac{r_{e} N_{b}}{\gamma} W_{s}(x, z)
$$

where $r_{e}$ is the classical electron radius and $N_{b}$ is the bunch population. The longitudinal wake is given by

$$
W_{s}(x, z)=\frac{1}{e} \int_{-\infty}^{\infty} \int_{-\infty}^{z-z_{i}} E_{s}\left(\frac{x-x^{\prime}}{\rho}, \frac{z-z^{\prime}}{2 \rho}\right) \lambda_{b}\left(x^{\prime}, z^{\prime}\right) d x^{\prime} d z^{\prime},
$$

where

$$
z_{i}=\rho\left[\phi-\beta \sqrt{\left(\frac{x-x^{\prime}}{\rho}\right)^{2}+4\left(1+\frac{x-x^{\prime}}{\rho}\right) \sin ^{2} \frac{\phi}{2}}\right],
$$

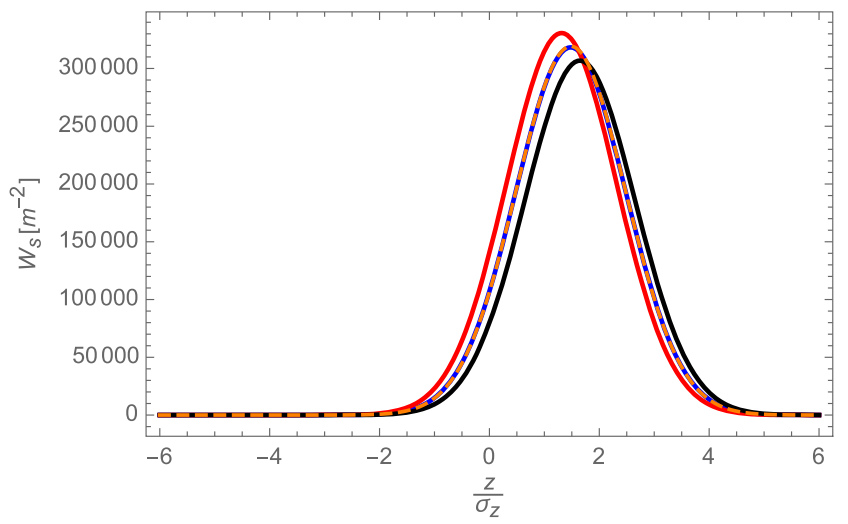

FIG. 3. The longitudinal wakes at various horizontal positions $x=2.5 \sigma_{x}$ (red), 0 (blue), and $-2.5 \sigma_{x}$ (black) at bending angle: $\phi=0.1 \mathrm{~m} / \rho$ and radius: $\rho=1.5 \mathrm{~m}$ for a round Gaussian bunch with $\sigma_{z}=\sigma_{x}=50 \mu \mathrm{m}$ and $\gamma=5000$. The dashed orange line represents Eq. (15) in the $1 \mathrm{D}$ theory.

and $\lambda_{b}$ is an arbitrary distribution that is normalized to $\iint \lambda_{b} d x d z=1$. The upper bound of the longitudinal integration is defined by $\eta=0$ as the entry point of the magnet. Here, $\phi=2 \alpha$ marks the angular position inside the magnet. The second integration of the horizontal coordinate properly takes account of the transverse dependency at the entry as we noted previously.

As a numerical example, we will use the same parameters in Ref. [8] in which the longitudinal transients were first studied. We will study a round Gaussian bunch with $\sigma_{z}=\sigma_{x}=50 \mu \mathrm{m}$ entering a magnet with a bending radius $\rho=1.5 \mathrm{~m}$. The longitudinal wakes with various horizontal offsets at the angular position $\phi=0.1 \mathrm{~m} / \rho$ are shown in Fig. 3. We plot the 1D theory [11],

$$
\frac{4}{\phi \rho} \lambda_{b}^{(1)}\left(z-\rho \frac{\phi^{3}}{6}\right)
$$

as the dashed orange line and find that it perfectly agrees to the on axis longitudinal wake. Here, $\lambda_{b}^{(1)}(z)$ is a longitudinal distribution normalized to 1 . The deviations due to the horizontal offsets are small but noticeable.

The horizontal force can be extracted from the equation of horizontal motion [16] and is given by $F_{x}=$ $e\left[E_{x}-\beta(1+\chi) B_{y}\right]$. It is essentially the Lorentz force differing with a factor $(1+\chi)$ multiplying magnetic field. This factor is due to the curvature of the coordinate system inside the bending magnet. Substituting Eqs. (9) and (11) for $E_{x}$ and $B_{y}$, respectively, into the force formula, we obtain 


$$
F_{x}=\frac{e^{2}\left\{\left(1+\beta^{2}\right)(1+\chi)-\left[1+\beta^{2}(1+\chi)^{2}\right] \cos 2 \alpha+(\eta-\beta \kappa) \sin 2 \alpha\right\}}{\gamma^{2} \rho^{2}\{\kappa-\beta[\eta+(1+\chi) \sin 2 \alpha]\}^{3}} .
$$

Similar to the longitudinal plane, using it as a Green function, we derive the change of the horizontal angle $x^{\prime}$ per unit length,

$$
x^{\prime \prime}=\frac{r_{e} N_{b}}{\gamma} W_{x}(x, z),
$$

where the horizontal bunch wake is given by

$W_{x}(x, z)=\frac{1}{e^{2}} \int_{-\infty}^{\infty} \int_{-\infty}^{z-z_{i}} F_{x}\left(\frac{x-x^{\prime}}{\rho}, \frac{z-z^{\prime}}{2 \rho}\right) \lambda_{b}\left(x^{\prime}, z^{\prime}\right) d x^{\prime} d z^{\prime}$.

The horizontal wakes with various horizontal offsets are shown in Fig. 4. Due to a partial cancellation between the electric and magnetic forces, the wake is much less than the one in the longitudinal plane. On the axis, the wake can be represented by

$$
\frac{2}{\rho} \lambda_{b}^{(1)}\left(z-\rho \frac{\phi^{3}}{6}\right)
$$

which is derived similarly to the longitudinal wake in the 1D theory at the limit of $\gamma \rightarrow \infty$ and is also plotted in dashed orange. It has the same function form as the longitudinal wake but with a reduction factor of $\phi / 2$. The deviations due to the horizontal offsets are not so large but again noticeable.

\section{CASE (B)}

Second, we consider the case when both the source and observation points are inside the magnet as illustrated

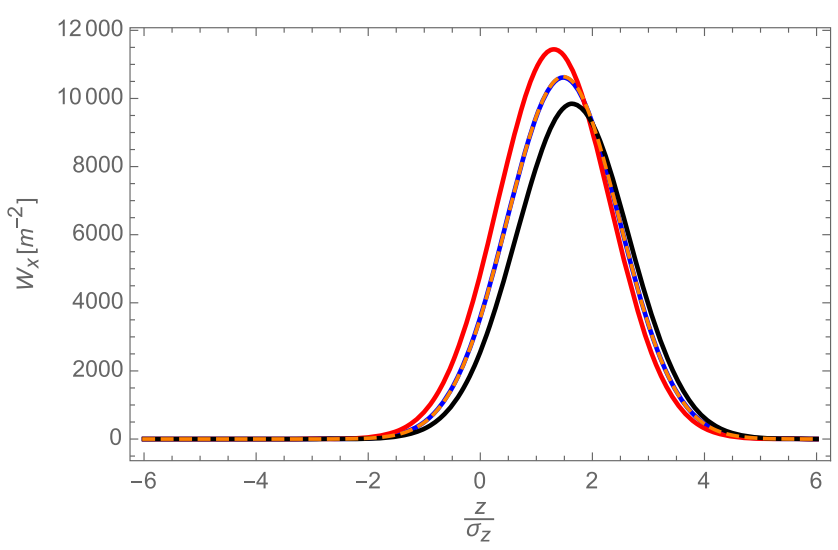

FIG. 4. The horizontal wakes at various horizontal positions $x=2.5 \sigma_{x}$ (red), 0 (blue), and $-2.5 \sigma_{x}$ (black) at bending angle: $\phi=0.1 \mathrm{~m} / \rho$ and radius: $\rho=1.5 \mathrm{~m}$ for a round Gaussian bunch with $\sigma_{z}=\sigma_{x}=50 \mu \mathrm{m}$ and $\gamma=5000$. The dashed orange line represents Eq. (19). in Fig. 5. This case has been studied in detail for the steady state in Ref. [16]. To avoid duplications, here we will focus on the modifications required from the transients.

Similar to the case (A), combining Eqs. (3) and (4), we can rewrite the retarded condition as

$$
\xi=\alpha-\frac{\beta \kappa}{2},
$$

where $2 \alpha$ is the retarded angle as illustrated in Fig. 5. After straightforward algebra, we find explicitly

$$
\kappa=\sqrt{\chi^{2}+4(1+\chi) \sin ^{2} \alpha} .
$$

For small angle $\alpha$, Eq. (20) can be casted into a quartic equation

$\alpha^{4}+\frac{3\left(1-\beta^{2}-\beta^{2} \chi\right)}{\beta^{2}(1+\chi)} \alpha^{2}-\frac{6 \xi}{\beta^{2}(1+\chi)} \alpha+\frac{3\left(4 \xi^{2}-\beta^{2} \chi^{2}\right)}{4 \beta^{2}(1+\chi)}=0$.

Since it has no third-order term, it is called the depressed quartic equation. Its analytical solution was discovered by Ferrari (1522-1565), who factorized the fourth-order polynomial by a product of two quadratic polynomials. The explicit solution of $\alpha$ is given in the Appendix of Ref. [16]. One way to estimate the validity of the quartic equation Eq. (22) is to compare $\alpha^{4}$ to the next highest order term $-2 \alpha^{6} / 15$, resulting in $\alpha \ll \sqrt{15 / 2}$.

Meanwhile, given $\chi$ and $\xi$, the angle $\alpha$ can also be obtained by direct Newton searching. A few numerical examples in the parameter region we are interested in are compared to the analytical solutions and shown in Fig. 6. Since the agreement between the analytic and numerical

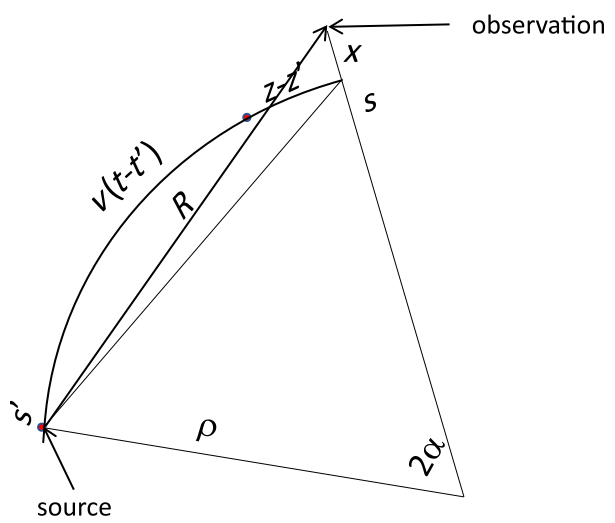

FIG. 5. A source particle moves on circular orbit with a bending radius $\rho$ and the observation point is also inside the magnet. 


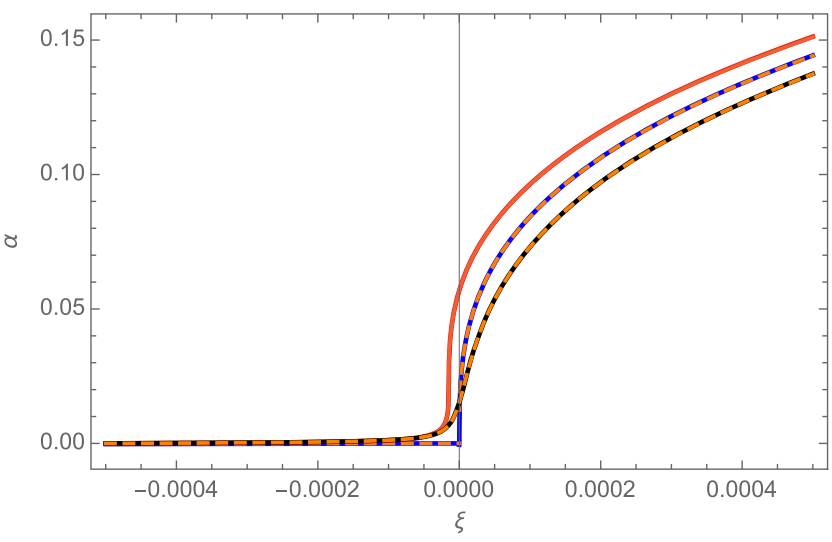

FIG. 6. Comparison of the numerical (solid line) and analytical (dashed orange) solutions of $\alpha$ as a function of $\xi$ with $\chi=0$ (blue), 0.001 (red), -0.001 (black), and $\gamma=5000$.

solutions is excellent, we will use the analytical solution in the rest of the paper for its computational efficiency.

Unlike the case (A), the source particle is in the bending region, acceleration $\dot{\vec{\beta}}$ in the Lienard-Wiechert formula in Eq. (1) generates the radiation often called the CSR fields. They become dominant at very high energy because of the suppression of $1 / \gamma^{2}$ in the space-charge fields. Since we are mostly interested in the ultrarelativistic case, we present here only the CSR fields for simplicity. Using trigonometry involving two triangles, we derive their nonvanishing field components,

$$
\begin{gathered}
E_{x}=\frac{e \beta^{2}\{\sin 2 \alpha[(1+\chi) \sin 2 \alpha-\beta \kappa]\}}{\rho^{2}[\kappa-\beta(1+\chi) \sin 2 \alpha]^{3}} \\
E_{s}=\frac{e \beta^{2}\{[\cos 2 \alpha-(1+\chi)][(1+\chi) \sin 2 \alpha-\beta \kappa]\}}{\rho^{2}[\kappa-\beta(1+\chi) \sin 2 \alpha]^{3}},
\end{gathered}
$$

$$
B_{y}=\frac{e \beta^{2}\{\kappa[(1+\chi) \sin 2 \alpha-\beta \kappa]\}}{\rho^{2}[\kappa-\beta(1+\chi) \sin 2 \alpha]^{3}} .
$$

Here again $\alpha$ is given by the solution of the retarded condition, through which the longitudinal dependency enters.

Similar to the case (A), we can use the longitudinal electric field as Green's function to compute the bunch wake,

$W_{s}(x, z)=\frac{1}{e} \int_{-\infty}^{\infty} \int_{z-z_{i}}^{z-z_{o}} E_{s}\left(\frac{x-x^{\prime}}{\rho}, \frac{z-z^{\prime}}{2 \rho}\right) \lambda_{b}\left(x^{\prime}, z^{\prime}\right) d x^{\prime} d z^{\prime}$

where $z_{i}$ is given by Eq. (14) and $z_{o}=-\beta\left|x-x^{\prime}\right|$. Due to a finite length of magnet, the retarded angle $2 \alpha$ is limited between $\phi$ and 0 , which defines the lower bound $z-z_{i}$ and the upper bound $z-z_{0}$ of the longitudinal integration correspondingly.

However, in this case, the source point can be infinitely close to the observation point. As a result, the Green's function can become highly singular and therefore practically useless for numerical computation of the bunch wakes. Instead, integration by parts are necessary to mitigate the singularity and make them integrable. In fact, it has been shown [16] that the longitudinal electric field $E_{s}$ in Eq. (24) can be written as

$$
E_{s}=\frac{e}{2 \rho} \frac{d w_{s}}{d \xi}
$$

in terms of the derivative of $\xi$ with

$$
w_{s}(\chi, \xi)=\frac{\beta^{2}\left(\cos 2 \alpha-\frac{1}{1+\chi}\right)}{\rho[\kappa-\beta(1+\chi) \sin 2 \alpha]} .
$$

Using Eq. (27) and integrating by parts, we rewrite the wake as

$$
\begin{aligned}
W_{s}(x, z)= & \int_{-\infty}^{\infty} \int_{z-z_{i}}^{z-z_{o}} w_{s}\left(\frac{x-x^{\prime}}{\rho}, \frac{z-z^{\prime}}{2 \rho}\right) \frac{\partial \lambda_{b}\left(x^{\prime}, z^{\prime}\right)}{\partial z^{\prime}} d x^{\prime} d z^{\prime} \\
& +\int_{-\infty}^{\infty} w_{s}\left(\frac{x-x^{\prime}}{\rho}, \frac{z_{i}}{2 \rho}\right) \lambda_{b}\left(x^{\prime}, z-z_{i}\right) d x^{\prime} \\
& -\int_{-\infty}^{\infty} w_{s}\left(\frac{x-x^{\prime}}{\rho}, \frac{z_{o}}{2 \rho}\right) \lambda_{b}\left(x^{\prime}, z-z_{o}\right) d x^{\prime}
\end{aligned}
$$

Two additional one-dimensional integrals are the results of the boundaries.

Numerical examples with the same parameters in the case (A) are shown in Fig. 7. Again on the axis, the wake agrees with the $1 \mathrm{D}$ theory [11],

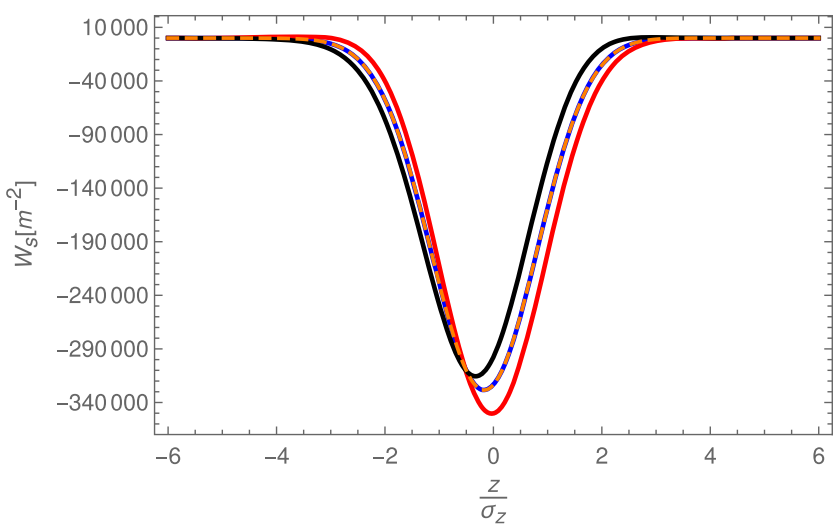

FIG. 7. The longitudinal wakes at various horizontal positions $x=2.5 \sigma_{x}$ (red), 0 (blue), and $-2.5 \sigma_{x}$ (black) at bending angle: $\phi=0.1 \mathrm{~m} / \rho$ and radius: $\rho=1.5 \mathrm{~m}$ for a round Gaussian bunch with $\sigma_{z}=\sigma_{x}=50 \mu \mathrm{m}$ and $\gamma=5000$. The dashed orange line represents Eq. (30) in the 1D theory. 
$-\frac{4}{\phi \rho} \lambda_{b}^{(1)}\left(z-\rho \frac{\phi^{3}}{24}\right)-\int_{z-\rho \frac{\phi^{3}}{24}}^{z} \frac{2}{3^{1 / 3} \rho^{2 / 3}\left(z-z^{\prime}\right)^{1 / 3}} \frac{\partial \lambda_{b}^{(1)}\left(z^{\prime}\right)}{\partial z^{\prime}} d z^{\prime}$, plotted in dashed orange. Similar to the case (A), the deviations due to the horizontal offsets are noticeable.

The horizontal wake $W_{x}(x, y)$ can be similarly written as the longitudinal one in Eq. (26) by replacing $w_{s}(\chi, \xi)$ with,

$$
\begin{aligned}
w_{x}(\chi, \xi)= & \frac{\beta^{2}}{\rho}\left\{\frac{1}{|\chi|(1+\chi)}\left[\left(2+2 \chi+\chi^{2}\right) F\left(\alpha, \frac{-4(1+\chi)}{\chi^{2}}\right)-\chi^{2} E\left(\alpha, \frac{-4(1+\chi)}{\chi^{2}}\right)\right]-\frac{\kappa\left[1-\beta^{2}(1+\chi) \cos 2 \alpha\right] \sin 2 \alpha}{\left[\kappa^{2}-\beta^{2}(1+\chi)^{2} \sin ^{2} 2 \alpha\right]}\right. \\
& \left.+\frac{\kappa^{2}-2 \beta^{2}(1+\chi)^{2}+\beta^{2}(1+\chi)\left(2+2 \chi+\chi^{2}\right) \cos 2 \alpha}{\beta(1+\chi)\left[\kappa^{2}-\beta^{2}(1+\chi)^{2} \sin ^{2} 2 \alpha\right]}\right\}-\frac{2}{\rho|\chi|} F\left(\alpha, \frac{-4(1+\chi)}{\chi^{2}}\right),
\end{aligned}
$$

where $F(\alpha, m)$ and $E(\alpha, m)=\int_{0}^{\alpha} \sqrt{1-m \sin ^{2} \theta} d \theta$ are the incomplete elliptic integrals of the first and second kind, respectively. The derivation can be found in Refs. [16] and [18]. It is worth noting that the last term is derived from the so-called potential term proposed [15] to cancel the centrifugal force.

Numerical examples with the same parameters in the longitudinal case is shown in Fig. 8. On the axis, the wake is given by,

$$
-\frac{4}{\rho} \lambda_{b}^{(1)}(z)
$$

plotted in dashed orange. It is the same result as the steadystate case and twice that found by Derbenev and Shiltsev [7].

\section{CASE (C)}

Third, we consider the case when both source and observation points are in drift spaces, one before entering and the other after exiting the magnet, respectively, as illustrated in Fig. 9.

Similar to the case (A), combining Eqs. (3) and (4), we can rewrite the retarded condition in terms of the distance $\eta$,

$$
\xi=\alpha+\frac{\lambda+\eta-\beta \kappa}{2}
$$

where $\lambda=L / \rho$ and $\alpha$ is a half of the bending angle $\phi_{m}$ of the magnet. After tedious algebra involving four triangles, we find explicitly

$$
\kappa=\sqrt{\lambda^{2}+\eta^{2}+\chi^{2}+4(1+\chi) \sin ^{2} \alpha+2[\lambda+(1+\chi) \eta] \sin 2 \alpha+2 \lambda \eta \cos 2 \alpha} .
$$

The retarded condition can be rewritten in terms of another more complicated quadratic equation,

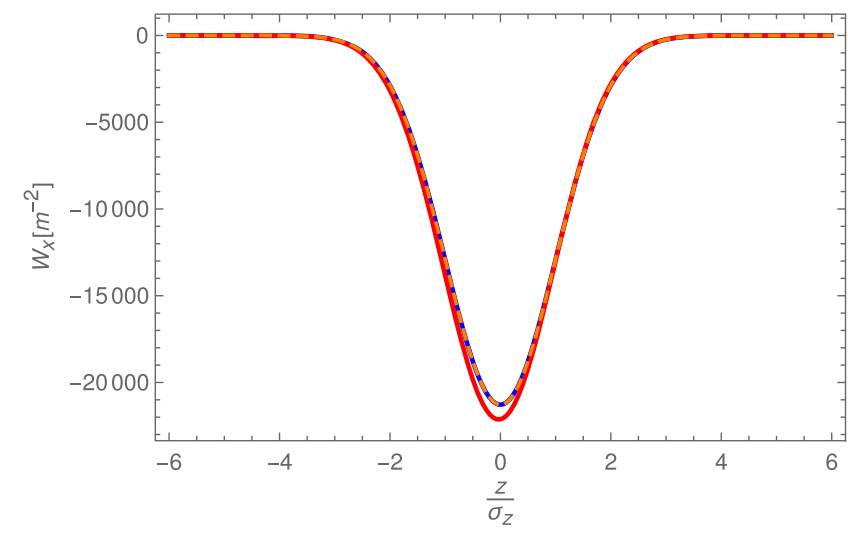

FIG. 8. The horizontal wakes at various horizontal positions $x=2.5 \sigma_{x}$ (red), 0 (blue), and $-2.5 \sigma_{x}$ (black) at bending angle: $\phi=0.1 \mathrm{~m} / \rho$ and radius: $\rho=1.5 \mathrm{~m}$ for a round Gaussian bunch with $\sigma_{z}=\sigma_{x}=50 \mu \mathrm{m}$ and $\gamma=5000$. The dashed orange line represents Eq. (32).

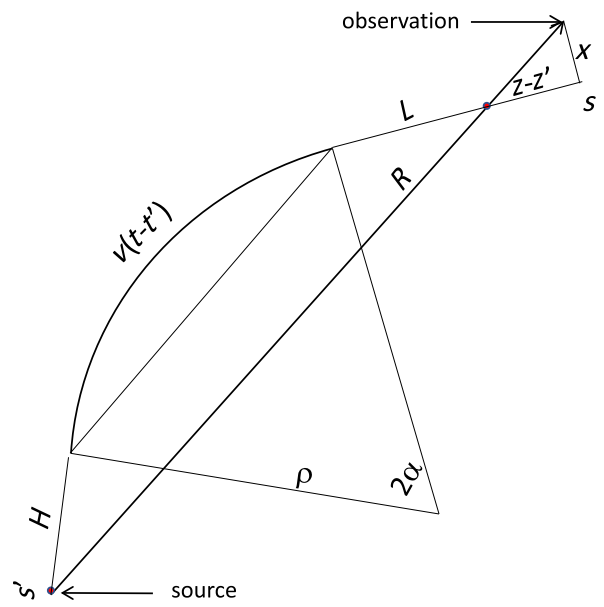

FIG. 9. A source particle moves on straight section before entering circle with radius $\rho$ while the observation point is in the free space exiting the magnet. 


$$
\begin{aligned}
& \frac{1}{4}\left(1-\beta^{2}\right) \eta^{2}+\left[\alpha-\xi+\frac{1}{2} \lambda-\frac{1}{2} \lambda \beta^{2} \cos 2 \alpha-\frac{1}{2} \beta^{2}(1+\chi) \sin 2 \alpha\right] \eta+\alpha^{2}+\alpha \lambda+\frac{1}{4}\left(1-\beta^{2}\right) \lambda^{2}-2 \alpha \xi-\lambda \xi \\
& \quad+\xi^{2}-\frac{1}{4} \beta^{2} \chi^{2}-\beta^{2}(1+\chi) \sin ^{2} \alpha-\frac{1}{2} \lambda \beta^{2} \sin 2 \alpha=0 .
\end{aligned}
$$

Here, $\eta$ is a root of the quadratic equation, formally also given by Eq. (8) with the coefficients defined by Eq. (35). It vanishes at

$\xi=\alpha+\frac{1}{2}\left(\lambda-\beta \sqrt{\lambda^{2}+\chi^{2}+4(1+\chi) \sin ^{2} \alpha+2 \lambda \sin 2 \alpha}\right)$,

which marks the entry point of the magnet.

We numerically search the retarded solutions using Eq. (33) and compare it to the analytical solutions for a short magnet relatively to the overtaking length $L_{0}$. The results are shown in Fig. 10, demonstrating prefect agreements. Naturally, we will use the analytical solutions for computing the bunch wakes.

Similar to the case (A), only the space-charge term in Eq. (1) contributes to the fields. After tedious algebra of solving four triangles consecutively, we derive the nonvanishing components,

$E_{x}=\frac{e[1+\chi-\cos 2 \alpha+(\eta-\beta \kappa) \sin 2 \alpha]}{\gamma^{2} \rho^{2}\{\kappa-\beta[\eta+\lambda \cos 2 \alpha+(1+\chi) \sin 2 \alpha]\}^{3}}$,
$E_{s}=\frac{e[\lambda+\sin 2 \alpha+(\eta-\beta \kappa) \cos 2 \alpha]}{\gamma^{2} \rho^{2}\{\kappa-\beta[\eta+\lambda \cos 2 \alpha+(1+\chi) \sin 2 \alpha]\}^{3}}$,

$B_{y}=\frac{e \beta[(1+\chi) \cos 2 \alpha-1-\lambda \sin 2 \alpha]}{\gamma^{2} \rho^{2}\{\kappa-\beta[\eta+\lambda \cos 2 \alpha+(1+\chi) \sin 2 \alpha]\}^{3}}$.

As a consistency check, we recover the fields in the case (A) while setting $\lambda=0$.

The longitudinal wake can be derived using the electric field $E_{s}$ in Eq. (38) as the Green's function. It can be written formally as Eq. (13), replacing $z_{i}$ with,

$$
z_{i}=\rho\left[\phi_{m}+\lambda-\beta \sqrt{\lambda^{2}+\left(\frac{x-x^{\prime}}{\rho}\right)^{2}+4\left(1+\frac{x-x^{\prime}}{\rho}\right) \sin ^{2} \frac{\phi_{m}}{2}+2 \lambda \sin \phi_{m}}\right] .
$$

The upper bound of the longitudinal integration $z-z_{i}$ is at the entry of the magnet where $\eta=0$.

Taking the same short magnet as a numerical example, we calculate the wakes at a position of $2 \mathrm{~cm}$ inside a $10-\mathrm{cm}$ long magnet with bending radius $\rho=1.5 \mathrm{~m}$ for a round

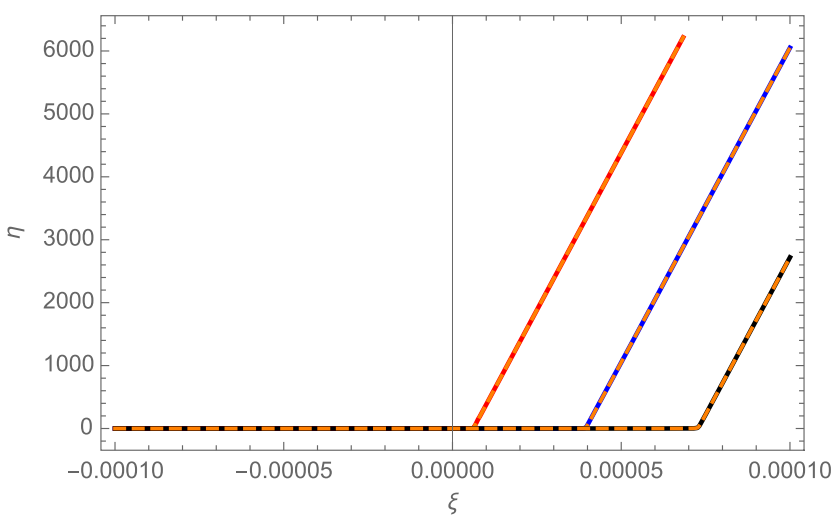

FIG. 10. Comparison of the numerical (solid line) and analytical (dashed orange) solutions of $\eta$ as a function of $\xi$ at $\lambda=0.02 \mathrm{~m} / \rho$, bending angle: $\phi_{m}=0.1 \mathrm{~m} / \rho$ and radius: $\rho=1.5 \mathrm{~m}, \chi=0$ (blue), 0.001 (red), -0.001 (black), and $\gamma=5000$.
Gaussian bunch with $\sigma_{z}=\sigma_{x}=50 \mu \mathrm{m}$. The longitudinal wakes at various horizontal offsets are shown in Fig. 11. We find that the on axis longitudinal wake agrees with the 1D theory [11],

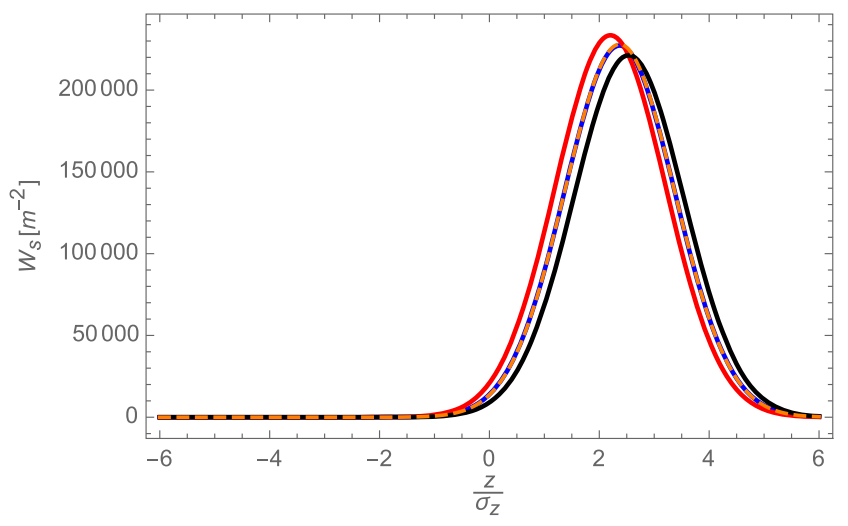

FIG. 11. The longitudinal wakes at various horizontal positions $x=2.5 \sigma_{x}$ (red), 0 (blue), and $-2.5 \sigma_{x}$ (black) at $\lambda=0.02 \mathrm{~m} / \rho$, bending angle: $\phi_{m}=0.1 \mathrm{~m} / \rho$ and radius: $\rho=1.5 \mathrm{~m}$ for a round Gaussian bunch with $\sigma_{z}=\sigma_{x}=50 \mu \mathrm{m}$ and $\gamma=5000$. The dashed orange line represents Eq. (41) in the 1D theory. 


$$
\frac{4}{\rho\left(\phi_{m}+2 \lambda\right)} \lambda_{b}^{(1)}\left(z-\rho \frac{\phi_{m}^{2}\left(\phi_{m}+3 \lambda\right)}{6}\right)
$$

which is plotted in dashed orange line.

Similar to the longitudinal wake, the horizontal wake can be written formally as Eq. (18) with the replaced integration bounds for this case. Naturally, the horizontal force, $F_{x}=e\left[E_{x}-\beta(1+\chi) B_{y}\right]$, should be updated according to Eqs. (37) and (39) for $E_{x}$ and $B_{y}$, respectively. It can be written as

$$
F_{x}=\frac{e^{2}\left\{\left(1+\beta^{2}\right)(1+\chi)-\left[1+\beta^{2}(1+\chi)^{2}\right] \cos 2 \alpha+\left[\eta-\beta \kappa+\beta^{2} \lambda(1+\chi)\right] \sin 2 \alpha\right\}}{\gamma^{2} \rho^{2}\{\kappa-\beta[\eta+\lambda \cos 2 \alpha+(1+\chi) \sin 2 \alpha]\}^{3}} .
$$

Again for checking, we recover the corresponding expression in Eq. (16) for the case (A) while setting $\lambda=0$.

We use the same parameters as the longitudinal case to compute the horizontal wakes with various horizontal offsets. The results are shown in Fig. 12. On the axis, the wake has the same shape as the longitudinal one but with a reduction factor of $\phi_{m} / 2$. It is given by

$$
\frac{2 \phi_{m}}{\rho\left(\phi_{m}+2 \lambda\right)} \lambda_{b}^{(1)}\left(z-\rho \frac{\phi_{m}^{2}\left(\phi_{m}+3 \lambda\right)}{6}\right),
$$

which is represented by the dashed orange color.

\section{CASE (D)}

Fourth, we consider the case when the source point is inside the magnet while the observation point is in drift spaces after exiting the magnet as illustrated in Fig. 13.

Similar to the case (B), combining Eqs. (3) and (4), we rewrite the retarded condition as

$$
\xi=\alpha+\frac{\lambda-\beta \kappa}{2}
$$

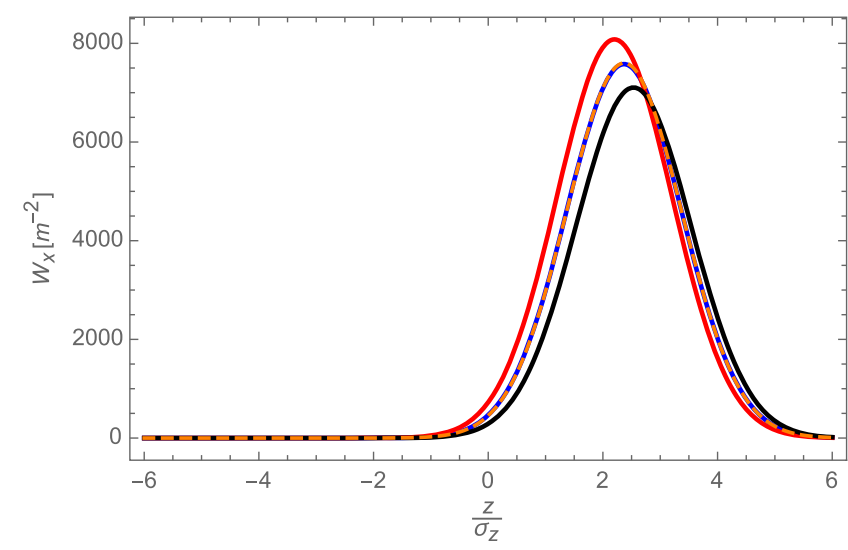

FIG. 12. The horizontal wakes at various horizontal positions $x=2.5 \sigma_{x}$ (red), 0 (blue), and $-2.5 \sigma_{x}$ (black) at $\lambda=0.02 \mathrm{~m} / \rho$, bending angle: $\phi_{m}=0.1 \mathrm{~m} / \rho$ and radius: $\rho=1.5 \mathrm{~m}$ for a round Gaussian bunch with $\sigma_{z}=\sigma_{x}=50 \mu \mathrm{m}$ and $\gamma=5000$. The dashed orange line represents Eq. (43). where $\alpha$ is a half of the retarded angle in the magnet. After straightforward algebra involving three triangles, we have explicitly

$$
\kappa=\sqrt{\lambda^{2}+\chi^{2}+4(1+\chi) \sin ^{2} \alpha+2 \lambda \sin 2 \alpha} .
$$

To find the analytic solution, we recast the retarded condition in Eq. (44) in terms of, to a fourth-order quartic equation of $\alpha$,

$$
\begin{aligned}
\alpha^{4} & +\frac{2 \lambda}{(1+\chi)} \alpha^{3}+\frac{3\left(1-\beta^{2}-\beta^{2} \chi\right)}{\beta^{2}(1+\chi)} \alpha^{2}+\frac{3\left(\lambda-\beta^{2} \lambda-2 \xi\right)}{\beta^{2}(1+\chi)} \alpha \\
+ & \frac{3\left(\lambda^{2}-\beta^{2} \lambda^{2}-4 \lambda \xi+4 \xi^{2}-\beta^{2} \chi^{2}\right)}{4 \beta^{2}(1+\chi)}=0 .
\end{aligned}
$$

For checking the consistency, we recover the depressed quartic equation in the case (B) while setting $\lambda=0$. However, here $\alpha$ is a root of the general quartic equation. The explicit solution is given in Appendix A.

Similar to the case (B), the angle $\alpha$ can also be found by direct numerical searching using Eq. (44). Several numerical examples in the parameter region we are interested in

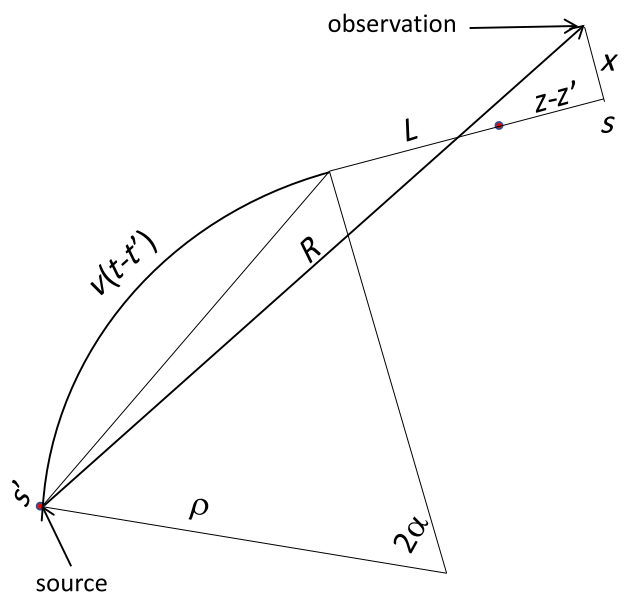

FIG. 13. A source particle moves on a circle with radius $\rho$ while the observation point is in the free space exiting the magnet. 


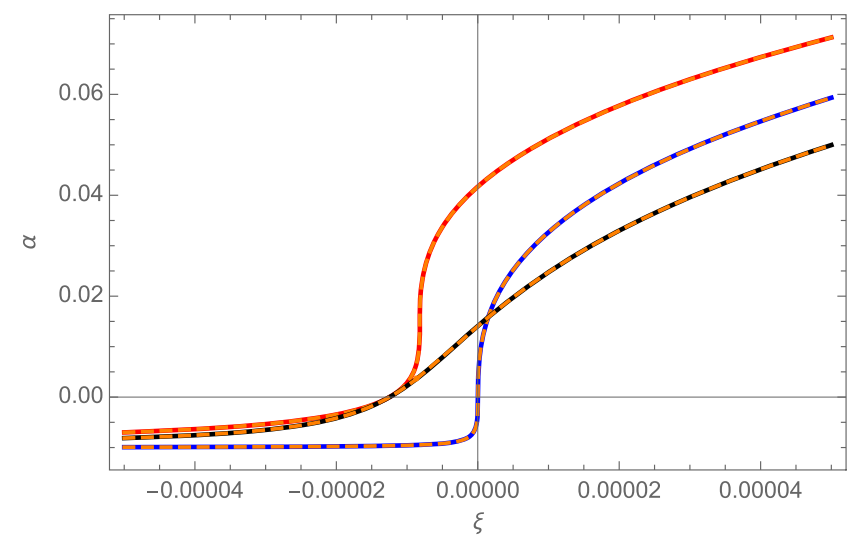

FIG. 14. Comparison of the numerical (solid line) and analytical (dashed orange) solutions of $\alpha$ as a function of $\xi$ with $\lambda=0.02, \chi=0$ (blue), 0.001 (red), -0.001 (black), and $\gamma=500$.

are compared to the analytical solutions and shown in Fig. 14.

Here we showed at $\gamma=500$ because the analytical solution becomes noisy at higher energy such as $\gamma=5000$ which were used in the previous cases. To avoid the noise, the numerical solution will be used in this case to compute the wakes. It is worth noting that $\alpha=0$ marks the exiting of the magnet.

Similar to the case (B), we ignore the space-charge fields with a focus on the high relativistic case and simplicity. After some algebra of calculating the CSR fields, we derive their nonvanishing components,

$E_{x}=\frac{e \beta^{2}(\lambda+\sin 2 \alpha)[\lambda \cos 2 \alpha+(1+\chi) \sin 2 \alpha-\beta \kappa]}{\rho^{2}\{\kappa-\beta[\lambda \cos 2 \alpha+(1+\chi) \sin 2 \alpha]\}^{3}}$,

$E_{s}=\frac{e \beta^{2}[\cos 2 \alpha-(1+\chi)][\lambda \cos 2 \alpha+(1+\chi) \sin 2 \alpha-\beta \kappa]}{\rho^{2}\{\kappa-\beta[\lambda \cos 2 \alpha+(1+\chi) \sin 2 \alpha]\}^{3}}$,

and

$$
B_{y}=\frac{e \beta^{2} \kappa[\lambda \cos 2 \alpha+(1+\chi) \sin 2 \alpha-\beta \kappa]}{\rho^{2}\{\kappa-\beta[\lambda \cos 2 \alpha+(1+\chi) \sin 2 \alpha]\}^{3}} .
$$

For checking, we recover the expressions for fields for the case (B) while setting $\lambda=0$.

Using the electric field $E_{s}$ in Eq. (48), the longitudinal wake can be written formally as Eq. (26), replacing $z_{i}$ with that in Eq. (40) and $z_{o}$ with

$$
z_{o}=\rho\left[\lambda-\beta \sqrt{\lambda^{2}+\left(\frac{x-x^{\prime}}{\rho}\right)^{2}}\right] .
$$

Because of a finite length of magnet, the retarded angle $2 \alpha$ is limited between $\phi_{m}$ and 0 , which defines the lower bound

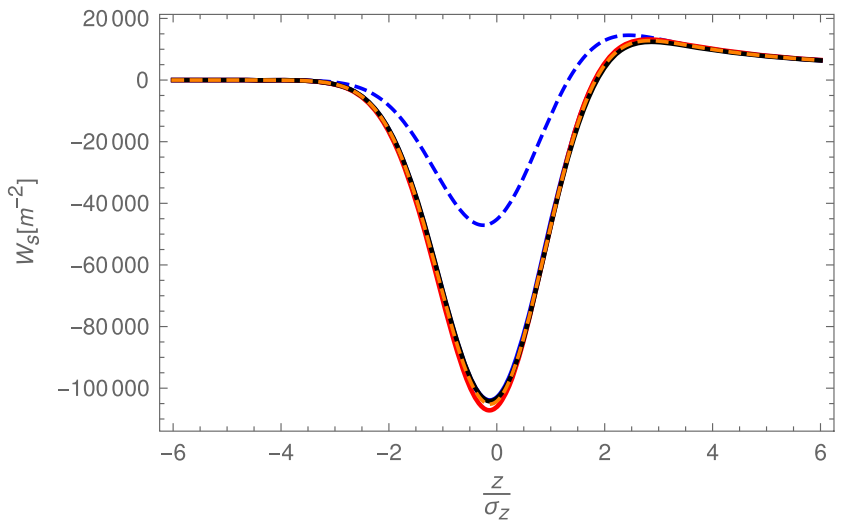

FIG. 15. The longitudinal wakes at various horizontal positions $x=2.5 \sigma_{x}$ (red), 0 (blue), and $-2.5 \sigma_{x}$ (black) at $\lambda=0.1 \mathrm{~m} / \rho$, bending angle: $\phi_{m}=0.5 \mathrm{~m} / \rho$ and radius: $\rho=1.5 \mathrm{~m}$ for a round Gaussian bunch with $\sigma_{z}=\sigma_{x}=50 \mu \mathrm{m}$ and $\gamma=5000$. The dashed orange line represents Eq. (51) in the 1D theory.

$z-z_{i}$ and the upper bound $z-z_{o}$ of the longitudinal integration, respectively.

Taking as a numerical example, we compute the wakes at a position of $10 \mathrm{~cm}$ exiting the magnet with bending radius $\rho=1.5 \mathrm{~m}$ for a round Gaussian bunch with $\sigma_{z}=\sigma_{x}=50 \mu \mathrm{m}$. The longitudinal wakes at various horizontal offsets are shown in Fig. 15.

To our surprise, the on axis wake plotted in the dashed blue line disagrees with the 1D theory [11],

$-\frac{4}{\rho}\left[\int_{z-\Delta z_{m}}^{z} \frac{1}{\phi+2 \lambda} \frac{\partial \lambda_{b}^{(1)}\left(z^{\prime}\right)}{\partial z^{\prime}} d z^{\prime}+\frac{1}{\phi_{m}+2 \lambda} \lambda_{b}^{(1)}\left(z-\Delta z_{m}\right)\right]$,

which is plotted in dashed orange. We find that an integral from the magnet exiting point to the position $\lambda \rho$ due to the space charge in free space is necessary to restore the agreement as shown in the solid blue line (covered by the solid red and black lines). The additional contribution can be attributed to the "regulation", namely subtracting the space-charge term: $e / \gamma^{2}\left(z-z^{\prime}\right)^{2}$ from the longitudinal electric field $E_{s}$ in the 1D theory. The calculation of the wakes in free space will be given in Appendix B. It is worth noting that the deviations due to the horizontal offsets are extremely small.

Similar to the longitudinal wake, we can write the horizontal wake as

$W_{x}(x, z)=\frac{1}{e^{2}} \int_{-\infty}^{\infty} \int_{z-z_{i}}^{z-z_{o}} F_{x}\left(\frac{x-x^{\prime}}{\rho}, \frac{z-z^{\prime}}{2 \rho}\right) \lambda_{b}\left(x^{\prime}, z^{\prime}\right) d x^{\prime} d z^{\prime}$,

with the same integration region. 
Here, the horizontal force is given by $F_{x}=e\left[E_{x}-\beta(1+\chi) B_{y}\right]-e \Phi / \rho$, where $\Phi$ is the Lienard-Wiechert potential. A derivation of the force can be found in Ref. [18]. Using Eqs. (47) and (49) for $E_{x}$ and $B_{y}$, respectively, we obtain

$$
F_{x}=\frac{e^{2} \beta^{2}[\lambda+\sin 2 \alpha-\beta(1+\chi) \kappa][\lambda \cos 2 \alpha+(1+\chi) \sin 2 \alpha-\beta \kappa]}{\rho^{2}\{\kappa-\beta[\lambda \cos 2 \alpha+(1+\chi) \sin 2 \alpha]\}^{3}}-\frac{e^{2}}{\rho^{2}\{\kappa-\beta[\lambda \cos 2 \alpha+(1+\chi) \sin 2 \alpha]\}} .
$$

Note that the second term is due to the potential.

We compute the horizontal wakes using the same parameters as the longitudinal case. The results including the additional contribution in the free space after the exiting are shown in Fig. 16. On the axis, the wake approximately is given by

$$
-\frac{1}{\rho} \lambda_{b}^{(1)}(z)
$$

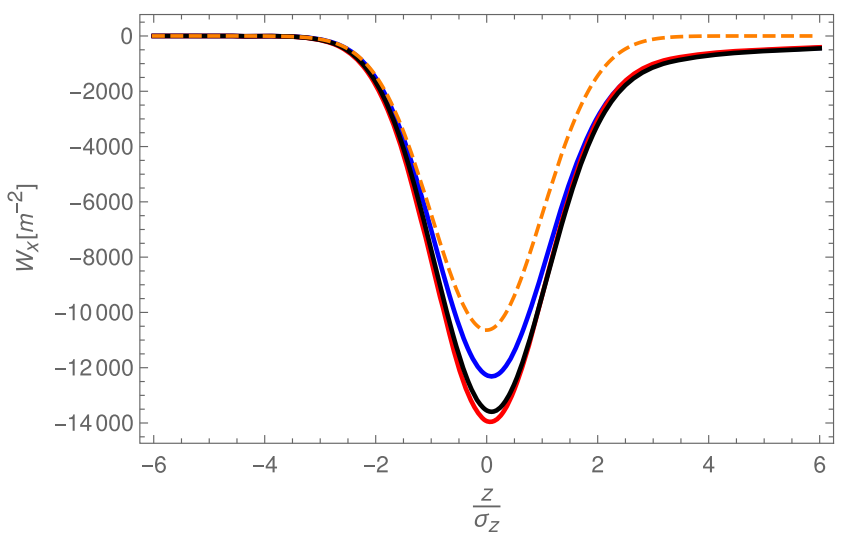

FIG. 16. The horizontal wakes at various horizontal positions $x=2.5 \sigma_{x}$ (red), 0 (blue), and $-2.5 \sigma_{x}$ (black) at $\lambda=0.1 \mathrm{~m} / \rho$, bending angle: $\phi_{m}=0.5 \mathrm{~m} / \rho$ and radius: $\rho=1.5 \mathrm{~m}$ for a round Gaussian bunch with $\sigma_{z}=\sigma_{x}=50 \mu \mathrm{m}$ and $\gamma=5000$. The dashed orange line represents Eq. (54). plotted in dashed orange. It is about a factor of 2 less than that found by Derbenev and Shiltsev [7].

\section{NUMERICAL EXAMPLES}

With all the relevant cases in hand, including the space charge in free space in Appendix B, we can compute the transient wakes after an electron bunch enters a magnet. The wakes are the summation of two cases, namely (A) and (B).

Here, we choose to use the same parameters in Ref. [8] from which the 1D theory was originated. With a round $50-\mu \mathrm{m}$ Gaussian bunch with $1 \mathrm{nC}$ charge entering a $50-\mathrm{cm}$ magnet with bending radius $\rho=1.5 \mathrm{~m}$, we compute the wakes and show them in Fig. 17.

For transient wakes after the bunch exits the magnet, the cases needed are (C) and (D), the space charge outlined in Appendix B. In this particular example, the magnet is much longer than the overtaking length $L_{0}$ and therefore there is no contribution from the case (C). We compute the wakes and depict them in Fig. 18.

We find that the longitudinal wakes on axis are identical to those in the 1D theory. The horizontal wakes display similar behalves as the longitudinal ones but at an order of magnitude smaller scale. Essentially, this explains why the horizontal wakes can be ignored in the 1D theory. It is worth noting that the transients are largest near the overtaking length $L_{0}=14 \mathrm{~cm}$.
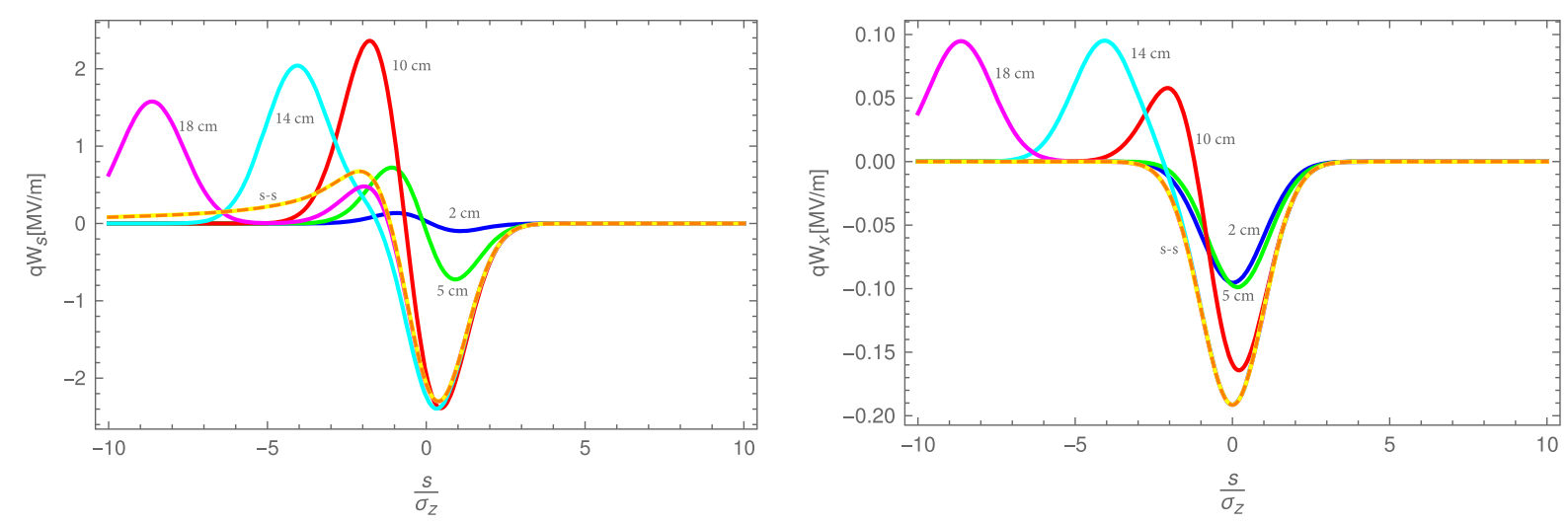

FIG. 17. The longitudinal (left) and the horizontal (right) wakes on axis at various positions after entering the magnet with $\gamma=5000$. 

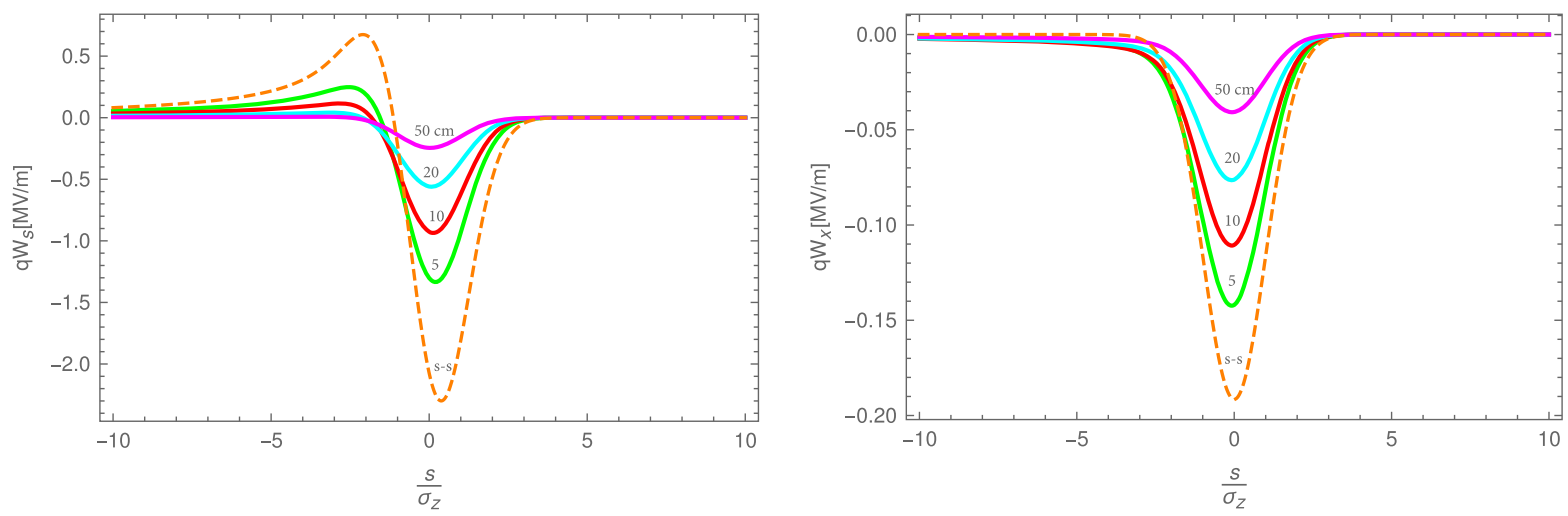

FIG. 18. The longitudinal (left) and the horizontal (right) wakes on axis at various positions after exiting the magnet with $\gamma=5000$.

\section{CONCLUSION}

A two-dimensional theory of coherent synchrotron radiation for realistic bending magnets including the transient effects is developed. The longitudinal wakes on axis agree precisely with those in the 1D CSR theory. Off axis, we see noticeable variations away from the on axis values. The horizontal wakes exhibit similar transient effects however at a much smaller scale.

The CSR is subtle because of singularity. In the twodimensional theory, the singularity has been properly treated with integration by parts in the cases where the source and the test particles can overlap with each other. As a result, the calculation of the bunch wakes become integrable. The computation is carried out with integration of the longitudinal derivative of bunch distribution. For the cases in which the two particles cannot approach each other infinitely closer, the conventional Green function with integration of the bunch distribution is sufficient.

Finally, we found that the "regularization" is not necessary in the two-dimensional theory. Instead, a new case, space charge in free space after exiting the magnet should be included for the wake after the bunch exits the magnet. Conceptually, the theory becomes straightforward and applicable to the transverse forces.

Naturally, extending this work to the three-dimensional theory [18] is the next step to complete the CSR theory. We have ignored the three-dimensional effects for a concern of a lengthy paper. Knowing that the vertical force is another order of magnitude smaller than the horizontal one, there are not much to lose by neglecting the vertical plane. It is worth noting that our theory does not include compression. It will be interesting to compare the theory with compression [19]. A systematic comparison to the different methods, including CSRTRACK [20], is underway.

\section{ACKNOWLEDGMENTS}

We would like to thank Y. Ding, Z. Huang, W. Lou, C. Mayes, G. Stupakov, G. White, and V. Yakimenko for many helpful and stimulating discussions. This work was supported by the Department of Energy under Contract No. DE-AC02-76SF00515.

\section{APPENDIX A: SOLUTION OF QUARTIC EQUATION}

For a quartic equation,

$$
x^{4}+b x^{3}+c x^{2}+d x+e=0
$$

the four roots are given by [21]

$$
\begin{aligned}
& x_{1,2}=-\frac{b}{4}-S \pm \frac{1}{2} \sqrt{-4 S^{2}-2 p+\frac{q}{S}} \\
& x_{3,4}=-\frac{b}{4}+S \pm \frac{1}{2} \sqrt{-4 S^{2}-2 p-\frac{q}{S}}
\end{aligned}
$$

where $p$ and $q$ are the coefficients of the second and the first degree in the associated depressed quartic, respectively,

$$
\begin{aligned}
& p=\frac{8 c-3 b^{2}}{8}, \\
& q=\frac{b^{3}-4 b c+8 d}{8},
\end{aligned}
$$

and

$$
\begin{aligned}
& S=\frac{1}{2} \sqrt{-\frac{2}{3} p+\frac{1}{3}\left(Q+\frac{\Delta_{0}}{Q}\right)}, \quad \text { and } \\
& Q=\sqrt[3]{\frac{\Delta_{1}+\sqrt{\Delta_{1}^{2}-4 \Delta_{0}^{3}}}{2}}
\end{aligned}
$$

with 


$$
\begin{aligned}
& \Delta_{0}=c^{2}-3 b d+12 e \text { and } \\
& \Delta_{1}=2 c^{3}-9 b c d+27 b^{2} e+27 d^{2}-72 c e .
\end{aligned}
$$

For the solution of Eq. (46), $\alpha=x_{1}$ if $x_{1}$ is real else $\alpha=x_{3}$.

\section{APPENDIX B: SPACE CHARGE IN FREE SPACE}

Here, we consider the case when the source point is in the free space after the exit of the magnet and the observation point is also outside of the magnet as illustrated in Fig. 19.

Given the velocity $v$ of a particle, the path length between point $s$ and $s^{\prime}$ can be written as

$$
s-s^{\prime}=v\left(t-t^{\prime}\right)+\left(z-z^{\prime}\right),
$$

where $z$ and $z^{\prime}$ are the longitudinal positions of the particle at time $t$ and $t^{\prime}$, respectively. Substituting $t-t^{\prime}$ in Eq. (3) into Eq. (B1), we obtain the retarded condition in terms of the retarded distance $L=s-s^{\prime}$,

$$
\xi=L-\beta \sqrt{x^{2}+L^{2}},
$$

where $\xi=z-z^{\prime}$. The solution of the retarded distance is given by

$$
L=\frac{\xi+\beta \sqrt{x^{2}\left(1-\beta^{2}\right)+\xi^{2}}}{1-\beta^{2}} .
$$

Similar to the cases (A) and (C), only the space-charge term in Eq. (1) contributes to the fields. After simple and straightforward algebra, we derive the nonvanishing components,

$$
\begin{gathered}
E_{x}=\frac{e x}{\gamma^{2}\left(\sqrt{x^{2}+L^{2}}-\beta L\right)^{3}}, \\
E_{s}=\frac{e\left(L-\beta \sqrt{x^{2}+L^{2}}\right)}{\gamma^{2}\left(\sqrt{x^{2}+L^{2}}-\beta L\right)^{3}}, \quad \text { and } \\
B_{y}=\frac{e \beta x}{\gamma^{2}\left(\sqrt{x^{2}+L^{2}}-\beta L\right)^{3}},
\end{gathered}
$$

where the retarded distance $L$ is given by Eq. (B3), through which the longitudinal dependency enters. Using Eqs. (B4)

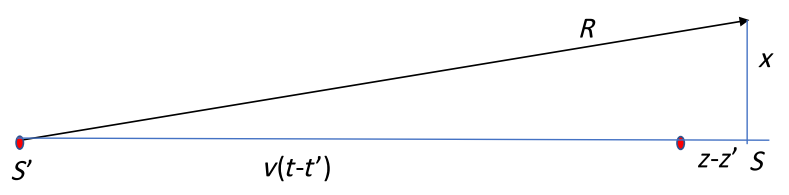

FIG. 19. A source particle moves on straight line with a constant velocity. and (B6), we obtain the Lorentz force $F_{x}=e\left(E_{x}-\beta B_{y}\right)$ and write it as

$$
F_{x}=\frac{e^{2} x}{\gamma^{4}\left(\sqrt{x^{2}+L^{2}}-\beta L\right)^{3}} .
$$

Since the source and observation points can overlap in this case, it is necessary to integrate by parts. Using the retarded condition in Eq. (B2), it is easy to show $E_{s}=e\left(d w_{s} / d \xi\right)$ and $F_{x}=e^{2}\left(d w_{x} / d \xi\right)$ with,

$$
\begin{aligned}
& w_{s}=-\frac{1}{\gamma^{2}\left(\sqrt{x^{2}+L^{2}}-\beta L\right)} \quad \text { and } \\
& w_{x}=-\frac{\beta x^{2}-\left(1-\beta^{2}\right) L \sqrt{x^{2}+L^{2}}}{\gamma^{2} x\left[x^{2}+L^{2}\left(1-\beta^{2}\right)\right]} .
\end{aligned}
$$

Applying them as kernels, the bunch wakes can be computed similarly to the case (B) with a different region of integration.

[1] J. Schwinger, On Radiation by Electrons in a Betatron (1945), (to be published) [transcribed by Miguel A. Furman in LBL Report No. LBL-39088, 1996].

[2] R. L. Warnock and P. Morton, Fields excited by a beam in a smooth toroidal chamber, Technical Report No. SLACPUB-5462, 1988.

[3] J. B. Murphy, S. Krinsky, and R. L. Gluckstern, Longitudinal wakefield for an electron moving on a circular orbit, Part. Accel. 57, 9 (1997).

[4] K. L. F. Bane, Y. Cai, and G. Stupakov, Threshold studies of the microwave instability in electron storage rings, Phys. Rev. ST Accel. Beams 13, 104402 (2010).

[5] S. Heifets, G. Stupakov, and S. Krinsky, Coherent synchrotron radiation instability in a bunch compressor, Phys. Rev. ST Accel. Beams, 5, 064401 (2002).

[6] Z. Huang and K.-J. Kim, Formulas for coherent synchrotron radiation microbunching in a bunch compressor chicane, Phys. Rev. ST Accel. Beams 5, 074401 (2002).

[7] Y.S. Derbenev, J. Rossbach, E. L. Saldin, and V.D. Shiltsev, Microbunch radiative tail-head interaction, DESY Print, Technical Report No. TESLA-FEL 95-05, 1995.

[8] M. Dohlus and T. Limberg, Emittance growth due to wake fields on curved bunch trajectories, Nucl. Instrum. Methods Phys. Res., Sect. A 393, 494 (1997).

[9] Y.S. Derbenev and V.D. Shiltsev, Transverse effects of microbunch radiative interaction, Technical Report No. SLAC-PUB-7181, 1996.

[10] E. L. Saldin, E. A. Schneidmiller, and M. V. Yurkov, On the coherent radiation of an electron bunch moving in arc of a circle, Nucl. Instrum. Methods Phys. Res., Sect. A 398, 373 (1997).

[11] G. Stupakov and P. Emma, CSR wake for a short magnet in ultrarelativistic limit, Technical Report No. SLAC-PUB9242, 2002. 
[12] M. Borland, Simple method for particle tracking with coherent synchrotron radiation, Phys. Rev. ST Accel. Beams 4, 070701 (2001).

[13] K. L.F. Bane et al., Measurements and modeling of coherent synchrotron radiation and its impact on the Linac Coherent Light Source electron beam, Phys. Rev. ST Accel. Beams, 12, 030704 (2009).

[14] R. Talman, Novel Relativistic Effect Important in Accelerator, Phys. Rev. Lett. 56, 1429 (1986).

[15] E. P. Lee, Cancellation of the centrifugal space-charge force, Part. Accel. 25, 241 (1990).

[16] Y. Cai, Coherent synchrotron radiation by electron moving on circular orbits, Phys. Rev. Accel. Beams 20, 064402 (2017).
[17] J. D. Jackson, Classical Electrodynamics, 3rd ed. (John Wiley \& Sons, New York, 1999).

[18] Y. Cai and Y. Ding, Three-dimensional effects of coherent synchrotron radiation by electrons in a bunch compressor, Phys. Rev. Accel. Beams 23, 014402 (2020).

[19] G. Stupakov and J. Tang, Calculation of the wake due to radiation and space charge forces in relativistic beams, Phys. Rev. Accel. Beams 24, 020701 (2021).

[20] M. Dohlus and T. Limberg, CSRtrack: Faster calculations of 3-D CSR effects, in Proceedings of FEL2004 Conference, Comitato Conferenze Elettra, Trieste, Italy (Joint Accelerator Conference Website, 2004), pp. 18-21.

[21] Quartic function, Wikipedia, the free encyclopedia. 